\section{Amount of surround and test-inducing separation in simultaneous brightness contrast*}

\author{
ROBERT E. COLE, Simon Fraser University, Vancouver, British Columbia, Canada \\ and \\ A. LEONARD DIAMOND, University of Hawaii, Honolulu, Hawaii 96822
}

Simultaneous brightness contrast is investigated in the fovea as a function of (1) amount of surround of the inducing field (Experiment 1) and (2) separation between the test and inducing fields (Experiment 2). Circular test and match fields subtending 14 min (radius) are used throughout. The inducing field, held constant in area, is a circular annulus (615 sq min) varying from a quadrant on one side of the test circle to an annulus completely surrounding the test circle. Test-field apparent brightness is not significantly affected by amount of inducing-field surround when the separation between centers of the test and inducing fields is held constant (Experiment 1). Experiment 2, though, shows that apparent brightness increases significantly as the separation between the centers of the test and inducing fields is increased.

The visual phenomenon referred to as simultaneous brightness contrast can occur when two visual fields are simultaneously presented to the eye. This phenomenon consists of a reported change in the brightness of one of the fields as a function of the presence of the second field.

Investigators have found several parameters that are important in determining the direction and degree of the contrast phenomena. For example, whether there is a brightness enhancement (increase) or a depression (decrease) of the test field (the field on which the brightness judgment is made) depends largely on the ratio of the luminance of the inducing field (the second field) to luminance of the test field. Brightness of the test field was found to be enhanced when the inducing luminance was less than the test luminance (Heinemann, 1955; Kleman et al, 1961) and depressed when the inducing luminance was greater than the test luminance (Diamond, 1953). In addition, depression of the test field was found to decrease with an increase in spatial separation (Beitel, 1936; Fry \& Alpern, 1953; Leibowitz, Mote, \& Thurlow, 1953) and increase with an increase in the inducing-field area (Diamond, 1955).

A theory of depression and enhancement in the brightness response has been developed by Diamond (1960) based on physiological-psychophysical assumptions and a number of empirical findings. The resulting mathematical mode] permits prediction of brightness contrast effects. Satisfactory prediction of contrast results, however, was possible only where the test and inducing fields were single adjacent rectangles. Functions obtained

* Rescarch supported in part by the National Science Foundation, Grant GB-5768, and the National Institutes of Health, Grant NB-06890. be greater for a given number of small inducers equally distributed around the perimeter of a test area than when these same inducers were adjacent.

However, the mean change of $.09 \log \mathrm{mL}$ found by Kaplan and Ripps was much less than the change that would be expected on the basis of the Diamond-Heinemann differences, or $2.30 \log \mathrm{mL}$. Since the Kaplan-Ripps study was at threshold, it would be instructive to determine what effect amount of surround has at suprathreshold levels.

The present study consists of two separate experiments. In both experiments a small foveally presented test field is used. The inducing field varies from a quadrant on one side of the test circle to a ring (of the same area) completely surrounding the test circle. In Experiment 1, test-field-inducing field separation, measured from the center of the test field to halfway between the inner and outer borders of the inducing annulus, is held constant. In Experiment 2 the test and inducing borders are held adjacent so that as the inducing field surrounds the test field more and more, test-field-inducing field separation decreases. The relative importance of surround and separation can be tested therefore in the same basic experimental design. as $2.30 \log \mathrm{mL}$ less, accord matween Diamond's a Heinemann's results) than a completely surrounding annulus of the sanc area and luminance, it has been postulated (Leibowitz et al, 1953; Diamond, 1960) that the amount by which the inducing field surrounds the test field is a powerful variable in determining brightness contrast effects. Support for such a postulate was found at threshold luminance by Kaplan and Ripps (1960). Depression was found to

\section{EXPERIMENT 1}

\section{Apparatus}

The apparatus, shown in Fig. 1, has separate light sources for the test and inducing fields (left path) and match field (right path). Each light source consists of a 150-W tungsten projection lamp, operated at $115 \mathrm{~V} \mathrm{ac}$ and $1.3 \mathrm{~A}$. The light passes through a section of heat-absorbing glass, a

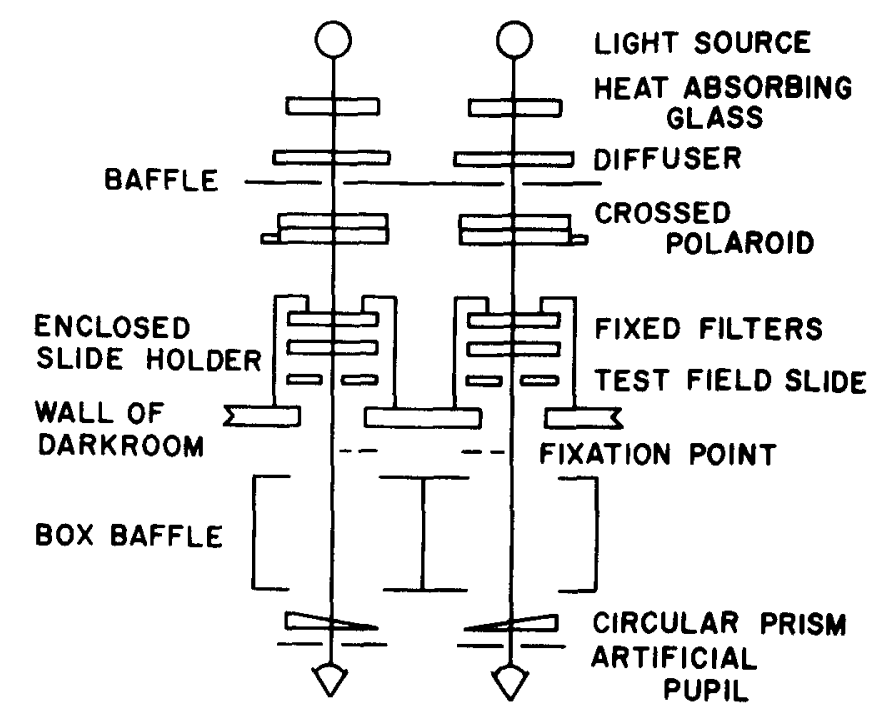

Fig. 1. Schema of the apparatus. 


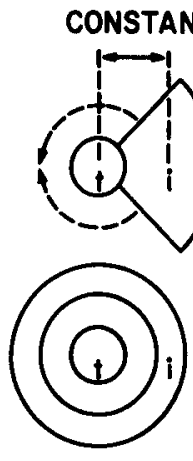

$\operatorname{Exp} 1$
VARIES

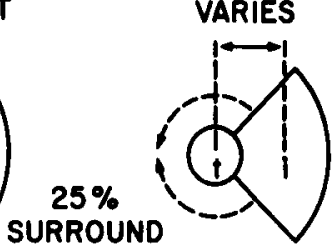

$100 \%$ SURROUND

$\operatorname{Exp} 2$
ALUMINUM

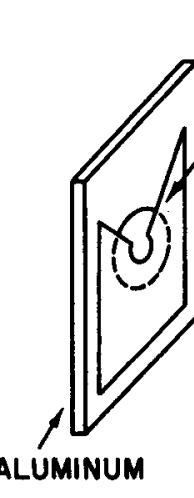

BLACK PAPER

FILTER

A

Fig. 2. (A) $25 \%$ and $100 \%$ surround patterns illustrating the difference in i-field variation between Experiment 1 and Experiment 2. (B) Schema of slide construction.

flashed opal glass diffuser, crossed Polaroids, and then Kodak neutral density Wratten filters. The Polaroids in the right path, operated by both the $E$ and the $S$, induce luminance of the match field over a calibrated range of 0.10 to $0.60 \mathrm{log}$ units. The Wratten filters are inserted in both visual pathways in order to control luminance values in large discrete steps. In the left visual path they change the testand inducing-field luminances simultaneously. In the right visual path they are used in conjunction with the variable Polaroid to adjust the luminance of the match field only.

The ratio of test luminance to inducing luminance is independently controlled with the use of additional neutral Wratten gelatin filters sealed between two pieces of $2 \times 2$ in. clear glass (see Fig. 2). A mask, made of thin aluminum sheet and black paper, is attached outside the glass slide to define a particular test-inducing pattern. A number of such masked slides were constructed, as described in Fig. 2, so that all the necessary combinations of test-inducing luminances and surround percentages were available. All three fields, test, inducing, and match, are held constant in area, each at $615 \mathrm{sq}$ min (measured in visual angle). ${ }^{1}$

Fixation points, $3 \mathrm{~min}$ in diam, are provided for each eye. Each fixation point has its own independent light source. For the right eye a point was located $15 \mathrm{~min}$ to the left of the match-field border, for the left eye $15 \mathrm{~min}$ to the right of the test-field border. Stimulation is essentially foveal in the binocular view, since the visual angle between the fused fixation point and the furthest edge of its adjacent pattern does not exceed $50 \mathrm{~min}$ (see Polyak, 1941).

A chin rest is provided to keep S's head steady and at a constant distance $(122 \mathrm{~cm})$ from the patterns. A center baffle insures that the left eye will see only the test and inducing patterns, and the right eye will see only the match pattern. Pupil size is held constant by $3-\mathrm{mm}$ artificial pupils mounted on optical trial frames worn by $\mathbf{S}$ during testing. The trial frames also contain circular prisms of $5 \mathrm{deg}$ which can be manipulated by $S$ to fuse the fixation points and thereby develop a binocular view.

\section{Procedure}

The apparatus is designed so that $\mathrm{S}$ can adjust the match field until it appears equal in brightness to the test field. In each experimental session, after a $15-\mathrm{min}$ period of dark adaptation in a light-tight cubicle, $S$ was instructed to: (1) put on the optician's trial frames, (2) place his chin on the chin rest, (3) fuse the fixation points by adjusting the prisms mounted on the trial frames, and (4) begin making matches. The $S$ then began making brightness equality matches following the psychophysical method of adjustment
(Guilford, 1954). By making at least four practice matches over a minimum 2-min period, $S$ was light-adapted to the pattern for that particular point. The values from the following eight matches were recorded as the experimental data. After a $2-\mathrm{min}$ rest period, followed by practice matches, $S$ made eight matches to the next test-inducing pattern. This procedure was repeated for five test-inducing patterns of different amounts of surround, with inducing-field luminance held constant during a given session.

Separation between the centers of the test and inducing fields was held constant at $18.48 \mathrm{~min}$, while the amount of surround was varied in four steps, from $0 \%$ (no inducing field) to $100 \%$. An additional point was taken at $100 \%$ surround with separation reduced to $11.28 \mathrm{~min}$ (test- and inducing-field borders adjacent). Luminance of inducing field was constant at $-0.22 \log \mathrm{mL}$ and its area was held at $615 \mathrm{sq}$ min. Luminance of the test field was constant at $-.42 \log \mathrm{mL}$ and its area at 615 sq min.

Three male college students with 20/20 vision according to the Snellen Chart test served as Ss for the experiment. All Ss received adequate training in making brightness-equality matches. Since stimulants have been found to affect brightness matching (Kleman et al, 1961), Ss were instructed to abstain from foods and beverages known to contain effective drugs during the $12 \mathrm{~h}$ period preceding the experiment.

\section{Results}

Table 1 lists the data separately for each of the three Ss. Each value represents the mean of 16 experimental matches, eight from each of two experimental days. The means of three Ss for each experimental point are graphed in Fig. 3, where the vertical dimension represents the match-field luminance (the measure of apparent brightness of the test field) and the horizontal dimension represents amount of surround of the inducing field.

The results of a two-way (Ss by Treatment) analysis of variance (see Table 2) show that the surround effect, tested over its interaction with Ss, is not significant $(p>.05, d f=2 / 4)$. In other words, the differences between mean

Table 2

Analysis of Variance of Match Luminance Values for Varying Amounts of Inducing-Field Surround

\begin{tabular}{lccccc}
\hline Percent Surround & $0 \%$ & $25 \%$ & $55 \%$ & $100 \%$ & $100 \%$ \\
\hline Separation (Minutes) & 18.48 & 18.48 & 18.48 & 18.48 & 11.28 \\
\hline Subjects & & & & & \\
KD & -.60 & -1.28 & -1.16 & -1.20 & -1.56 \\
BY & -.67 & -.96 & -.96 & -.98 & -1.22 \\
AE & -.08 & -.64 & -.62 & -.74 & -1.02 \\
\hline
\end{tabular}

\begin{tabular}{|c|c|c|c|}
\hline Source & df & Mean Square & $\mathrm{F}$ \\
\hline Surround (A) & 2 & .050 & 1.25 \\
\hline Subjects (B) & 2 & 3.644 & $91.10^{* * *}$ \\
\hline$A \times B$ & 4 & .040 & $4.34 * *$ \\
\hline Error (w) & 135 & .009 & \\
\hline
\end{tabular}




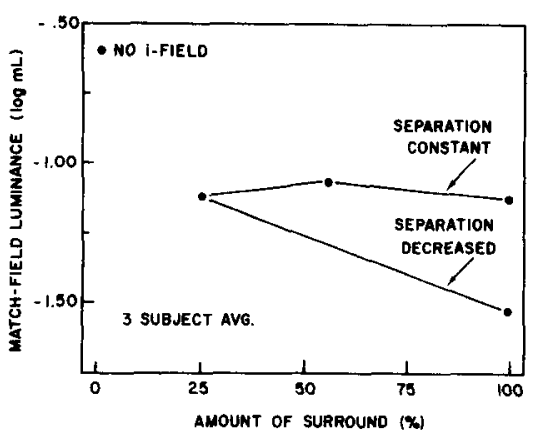

Fig. 3. Match luminance as a function of the percentage of the test-field border that is covered by the inducing-field (amount of surround) for two separations.

settings of the match field $\left(B_{m}\right)$ for the $25 \%, 55 \%$, and $100 \%$ surround conditions with separation held constant, are not greater than differences that would be expected to occur due to chance. On the other hand, when test-inducing-field separation is reduced as the amount of surround is increased, apparent brightness is depressed. Using the A by B interaction as the error term, the difference between the 11.28-min separation pattern and each of the $18.48-\mathrm{min}$ separation patterns was significant $(\mathrm{p}<.01$ in each case). Thus, the results indicate that variation in amount of surround is only detectable if the variable of test-inducing-field separation is varied simultaneously.

\section{EXPERIMENT 2}

\section{Apparatus}

The apparatus is the same as in Experiment 1, except for a change in construction of the slides. The slides were constructed with test- and inducing-field near borders always superimposed (see Fig. 2) with the result that as amount of surround increased, the separation between inducing and test field (as measured above) decreased from $18.48 \mathrm{~min}$ (at $25 \%$ surround) to $11.28 \mathrm{~min}$ (at $100 \%$ surround).

\section{Procedure}

The procedure was identical to that of
Experiment 1, with the exception that separation was varied in seven steps within any one session. Luminance of the test field was held constant at $-.42 \log \mathrm{mL}$ and the luminance of the inducing field was varied from $-\infty$ to $-.22 \log \mathrm{mL}$. The three Ss are the same as in Experiment 1.

\section{Results}

The results of three Ss are presented in Table 3. In Fig. 4 the mean match luminance for three $S s$ is plotted against the separation between centers of the test and inducing fields. It appears from Fig. 4 that test brightness increases with an increase in separation between the centers of the fields. This holds true for all inducing-field luminances except the $-\infty \log \mathrm{mL}$ luminance. It also appears that the rate of change increases with increased inducing-field luminance.

Table 4, which lists the $F$ tests resulting from a four-way, mixed-model analysis of variance, supports the above visual interpretation of Fig. 4, since the $\mathrm{F}$ ratios of the main effects (separation and luminance) over their respective interactions with $\mathrm{Ss}$ are significant. The significant Separation by, Luminance (A by B) interaction indicates that the amount of effect caused by increasing separation is dependent on the luminance level of the inducing field.

\section{DISCUSSION}

The purpose of the present study was to determine the effects of varying amounts of surround of an inducing field on suprathreshold brightness judgments. The results of Experiment 1 (see Fig. 3) show that when separation between centers of the lields is held constant, increasing the amount of surround of the inducing field (of constant area) does not result in a measurably significant change in the suprathreshold brightness response.

As was pointed out above, however, Kaplan and Ripps (1960) found that an inducing area produces slightly more brightness depression $(.09 \log \mathrm{mL})$ when spread around a test field than when concentrated on one side. The measure of brightness used was absolute threshold luminance.

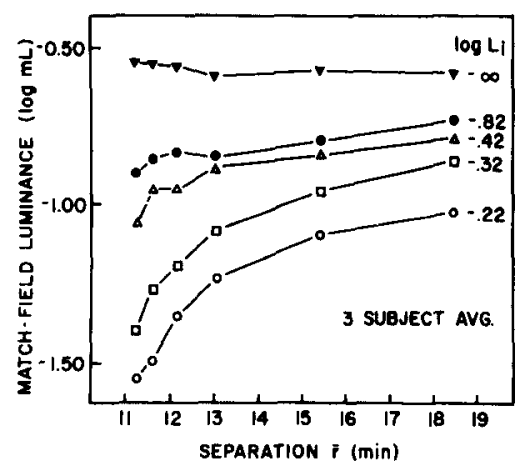

Fig. 4. Match luminance as a function of separation between centers of the $t$ and $i$ fields and $i$-field luminance.

The discrepancy between the results of the present study and the Kaplan-Ripps study could be attributed to the fact that at suprathreshold luminances and under foveal stimulation, amount of surround as a variable is even less important (to the extent of being insignificant) than at threshold. In any case, the discrepancy is a small one, and we can conclude that for the conditions of our Experiment 1, amount of surround is not an important variable.

Experiment 2, on the other hand, brings out the fact that the important basic variable when amount of surround is changed seems to be test-field-inducing field separation. That is, if an inducing field of constant area always borders upon a test field, it will change in effective separation from a test field as it changes from an adjacent to a surrounding field. This is because the average distance of the receptor elements in the inducing (retinal) field changes with respect to the elements in the test field.

\section{SUMMARY AND CONCLUSIONS}

Simultaneous brightness contrast is investigated in the fovea as a function of (1) amount of surround of the inducing field (Experiment 1) and (2) separation between the test and inducing fields (Experiment 2).

Circular test and match fields subtending 14 min (radius) are used throughout. The

Table 3

Match-Field Luminance ( $\log \mathrm{mL}$ ) as a Function of Separation Between Centers of the Test and Inducing Fields for Five Inducing Luminances (The test-field luminance is held at $-.42 \log \mathrm{mL}$.)

\begin{tabular}{|c|c|c|c|c|c|c|c|c|c|c|c|c|c|c|c|}
\hline \multirow{3}{*}{$\begin{array}{c}\text { Separation } \\
\mathbf{r} \text { (Min) }\end{array}$} & \multicolumn{15}{|c|}{$\log L_{i}$} \\
\hline & \multicolumn{3}{|c|}{$-\infty$} & \multicolumn{3}{|c|}{-.82} & \multicolumn{3}{|c|}{-.42} & \multicolumn{3}{|c|}{-.32} & \multicolumn{3}{|c|}{-.22} \\
\hline & $\mathrm{KD}$ & $\mathrm{BY}$ & $\mathrm{AE}$ & $\mathrm{KD}$ & BY & $\mathrm{AE}$ & $\mathrm{KD}$ & BY & $\mathrm{AE}$ & $\mathrm{KD}$ & BY & $\mathbf{A E}$ & KD & BY & $\mathrm{AE}$ \\
\hline $18.48 \mathrm{~min}$ & -.57 & -.61 & -.55 & -.88 & -.72 & -.58 & -1.02 & -.72 & -.64 & -1.07 & -.76 & -.75 & -1.29 & -.86 & -.90 \\
\hline $15.48 \mathrm{~min}$ & -.57 & -.61 & -.54 & -.99 & -.84 & -.56 & -1.10 & -.70 & -.71 & -1.22 & -.82 & -.84 & -1.39 & -.90 & -.99 \\
\hline $13.02 \mathrm{~min}$ & -.62 & -.59 & -.55 & -1.09 & -.84 & -.61 & -1.10 & -.79 & -.75 & -1.41 & -.93 & -.91 & -1.52 & -1.30 & -1.15 \\
\hline $12.18 \mathrm{~min}$ & -.50 & -.63 & -.55 & -1.05 & -.86 & -.58 & -1.25 & -.82 & -.79 & -1.53 & -1.02 & -1.02 & -1.57 & -1.13 & -1.36 \\
\hline $11.58 \mathrm{~min}$ & -.54 & -.62 & -.50 & -1.07 & -.87 & -.60 & -1.28 & -.75 & -.82 & -1.65 & -1.09 & -1.07 & -1.64 & -1.22 & -1.60 \\
\hline $11.28 \mathrm{~min}$ & -.51 & -.65 & -.49 & -1.16 & -.87 & -.66 & -1.44 & -.85 & -.88 & -1.76 & -1.19 & -1.24 & -1.62 & -1.25 & -1.78 \\
\hline
\end{tabular}


Table 4

Results of a Four-Way Mixed Model Analysis of Variance

\begin{tabular}{|c|c|c|c|}
\hline Source & df & Mean Square & $\mathrm{F}$ \\
\hline Separation (A) & 6 & 6.723 & $22.40 * * *$ \\
\hline Luminance (B) & 4 & 19.474 & $11.94 * * *$ \\
\hline Subject (C) & 2 & 13.607 & $45.33^{* * *}$ \\
\hline Replication (D) & 1 & .335 & 1.42 \\
\hline$A \times B$ & 24 & .869 & $6.99 * * *$ \\
\hline$A \times C$ & 12 & .300 & $2.41^{*}$ \\
\hline$A \times D$ & 6 & .056 & 1.10 \\
\hline $\mathrm{B} \times \mathrm{C}$ & 8 & 1.632 & $3.71 *$ \\
\hline$B \times D$ & 4 & .736 & 1.67 \\
\hline $\mathrm{C} \times \mathrm{D}$ & 2 & .236 & .54 \\
\hline$A \times B \times C$ & 48 & .124 & $2.28 *$ \\
\hline$A \times B \times D$ & 24 & .016 & .29 \\
\hline$A \times C \times D$ & 12 & .051 & .93 \\
\hline$B \times C \times D$ & 8 & .439 & $8.05^{* * *}$ \\
\hline$A \times B \times C \times D$ & 48 & .055 & $8.46 * * *$ \\
\hline Error (w) & 1470 & .006 & \\
\hline Total & 1679 & 1.249 & \\
\hline
\end{tabular}

${ }^{*} p=.05, * * p=.01, * * p=.001$

inducing field, held constant in area, is a circular annulus (615 sq $\mathrm{min}$ ) varying from a quadrant on one side of the test circle to an annulus completely surrounding the test circle. A binocular matching technique is used in both experiments.

The results of Experiment 1 show no significant change in test-field apparent brightness as a function of amount of inducing-field surround when the separation between centers of the test and inducing fields is held constant. Consequently, in Experiment 2, separation between test- and inducing-field centers is varied by varying amount of surround, and the results are attributed mainly to changes in separation between centers of the fields.
Psychological Review, 1960, 67, 168-199.

FRY, G. A., \& ALPERN, M. The effect of a peripheral glare source upon the apparent brightness of an object. Journal of the Optical Society of America, 1953, 43, 189-195.

GUILFORD, J. P. Psychometric methods New York: McGraw-Hill, 1954.

HARTLINE, H. K., \& RATLIFF, F. Inhibitory interaction of receptor units in the eye of Limulus. Journal of General Physiology, 1957, 40, 357-376.

HEINEMANN, E. G. Simultaneous brightness induction as a function of inducing- and test-field luminance. Joumal of Experimental Psychology, 1955, 50, 89-96.

KAPLAN, I. T. \& RIPPS, H. Effect on visual threshold of light outside the test area. Joumal of Experimental Psychology, 1960, 60, 284-289.

KLEMAN, J. P., DIAMOND, A. L., \& SMITH, E. Effects of caffeine on enhancement in foveal simultaneous contrast. Journal of Experimental Psychology, 1961, 61, 18-22.

LEIBOWITZ, H., MOTE, F. A., \& THURLOW, W. R. Simultaneous contrast as a function of separation between test and inducing fields. Journal of Experimental Psychology, 1953, 46, 453-456.

The results of Experiment 2 show that test-field apparent brightness increases significantly with an increase in separation. Rate of brightness change increases with increases in inducing-field luminance.

\section{REFERENCES}

BEITEL, R. 1. Inhibition of threshold excitation in the human eye. Journal of General Psychology, 1936, 14, 31-36.

DIAMOND, A. L. Foveal simultaneous brightnes contrast as a function of inducing. and test-field luminance. Journal of Experimental Psychology, 1953, 45, 305-414.

DIAMOND, A. L. Foveal simultaneous contrast as a function of inducing-field area. Journal of Experimental Psychology, 1955, 50, 144-152.

DIAMOND, A, L. Theory of depression and enhancement in the brightress response.
LINDQUIST, E. F. Design and analysis of experiments in psychology and education. Cambridge: Riverside Press, 1953.

POLYAK, S. L. The retina. Chicago: University of Chicago Press, 1941.

\section{NOTE}

1. Owing to the difficulty of constructing small patterns, a random variation of less than $8 \%$ accurred in the inducing area. According to Diamond's results (1955), however, the area change, at the luminances studied in the present investigation, produces an insignificant amount of change in the brightness response.

(Accepted for publication May 28, 1970.) 